\title{
Strates
}

STRATES Matériaux pour la recherche en sciences sociales

Hors-série | 2002

Parcours dans la recherche urbaine, Michel Rochefort, un géographe engagé

\section{Mode de production technico-scientifique et différenciation spatiale}

\section{Milton Santos}

\section{(2)enEdition}

\section{Journals}

Édition électronique

URL : http://journals.openedition.org/strates/536

DOI : $10.4000 /$ strates.536

ISSN : $1777-5442$

Éditeur

Laboratoire Ladyss

Édition imprimée

Date de publication : 1 janvier 2002

ISSN : 0768-8067

Référence électronique

Milton Santos, « Mode de production technico-scientifique et différenciation spatiale », Strates [En ligne], Hors-série | 2002, mis en ligne le 02 mai 2005, consulté le 08 septembre 2020. URL : http:// journals.openedition.org/strates/536 ; DOI : https://doi.org/10.4000/strates.536

Ce document a été généré automatiquement le 8 septembre 2020

Tous droits réservés 


\title{
Mode de production technico- scientifique et différenciation spatiale
}

\author{
Milton Santos
}

\section{NOTE DE L'ÉDITEUR}

Traduit du portugais par Marie-Pierre Mazéas

1 Désigné par Marx et Engels, comme point nodal de leur théorie de l'histoire, le terme de mode de production n'a pas reçu de véritable définition de ses fondateurs. Sans doute est-ce pour cette raison que ce concept fait l'objet de multiples controverses, auxquelles ont été mêlés marxistes et non-marxistes, surtout dans les années soixante et soixante-dix, avant qu'il ne tombe en relative désuétude, tout du moins parmi les géographes. Quant au mot espace, dont découle l'expression différenciation spatiale, il a été employé avec une très grande imprécision dans le vocabulaire de la géographie. Comment rapprocher ces deux termes ambigus dans une tentative d'interprétation géographique du monde actuel ? Cette question exige une clarification des concepts.

Clarification des concepts

2 Pour Marx, la notion de mode de production est centrale, car à chaque étape d'évolution de la société, les hommes prennent part à un processus unitaire, qui inclut les formes matérielles de production, les relations des hommes entre eux et avec les choses (naturelles et artificielles) et leur propre façon d'être, c'est-à-dire la manière dont ils expriment leur vie.

On semble s'accorder sur le fait que la vie sociale, prise comme un tout, se caractérise par l'incessante rénovation des formes productives et des relations de production, autrement dit, des modes de production. Chaque mode de production constitue une étape dans la production de l'histoire et se manifeste par l'apparition de nouveaux outils de travail et de nouvelles pratiques sociales. Comme produire et produire de 
l'espace sont synonymes, à chaque nouveau mode de production (ou à chaque nouveau moment d'un même mode de production) la structure et le fonctionnement de l'espace changent. L'espace peut être défini comme un ensemble indissociable de systèmes d'objets (outils du travail) et de systèmes d'actions (pratiques sociales). Modes de production et espace géographique évoluent ensemble, mus par une logique unitaire.

4 À l'aube des temps historiques, modes de production et formations économico-sociales ne faisaient qu'un. L'action des modes de production sur l'espace se faisait pratiquement sans médiations.

5 À partir du XVI e siècle, avec l'expansion du capitalisme, apparaît la possibilité de vastes échanges, intercontinentaux et transocéaniques, de plantes, d'animaux et d'hommes, avec leurs façons d'être et leurs savoir-faire. Des modes de production jusque-là distincts tendent à converger, et les aires concernées par ce mouvement unificateur font, de ce point de vue, l'expérience d'une histoire commune. Ce sont les « économiesmonde », de Fernand Braudel. La progression du capitalisme marque un élargissement et un approfondissement de cette logique, en incluant chaque fois un plus grand nombre de sociétés et de territoires. Le mode de production capitaliste tend à être unique.

6 Mais l'action de ce mode de production sur les différents territoires passe par la médiation des formations sociales constituées sous l'égide de l'État national.

7 Avec la globalisation, peut-on aujourd'hui penser que, de nouveau, le processus spatial serait une émanation directe du mode de production? Ceux qui croient en la mort de l'État et à la fin des frontières diront que oui. En vérité, la médiation de l'État, de la société civile, et de la configuration territoriale elle-même reçue en héritage sont des données fondamentales pour expliquer l'impact variable du mode de production sur les différents pays et régions. Mais il est également certain qu'à aucune autre époque, un mode de production n'a connu une diffusion aussi généralisée et n'a eu une prégnance aussi profonde et efficace, aux quatre coins de la terre. Ce mode de production global est un élément d'explication majeur de la réalité géographique actuelle.

Le phénomène technique et l'intelligence planétaire

8 Comprise comme l'ensemble des réalités et phénomènes contemporains qui distinguent l'époque actuelle des périodes antérieures, la globalisation peut être prise comme un véritable paradigme.

9 Ce point de départ peut susciter des objections : la globalisation est incomplète, elle se fait de façon inégale, elle est perverse, elle n'instaure pas de nouvelle période; elle constitue seulement un prolongement de la phase antérieure. De ce fait, les conditions ne seraient pas réunies pour qu'elle puisse servir de paradigme.

10 Certes, la globalisation est incomplète. Mais quelle autre époque de l'histoire s'est manifestée de façon aussi homogène sur toute la face de la terre ou a réussi à arrêter le progrès?

11 Les époques se succèdent sans interruption, et en même temps qu'elles s'imposent, elles conservent des vestiges du passé. Quelques-uns des nouveaux vecteurs connaissent une diffusion plus rapide et plus étendue (c'est le cas actuel de l'information), tandis que d'autres se propagent plus lentement et de façon plus sélective (c'est le cas des mentalités). Renforçant les distorsions et les inégalités, créant de nouvelles formes de dépendance et de pénurie, le processus actuel de globalisation est également pervers. Mais il est un fait que le mouvement d'internationalisation, 
instauré avec le capitalisme commercial, a atteint un stade suprême, avec le capitalisme technologique globalisé.

Le monde structuré comme un tout, devient « uni » (R. Robertson, 1990). Il constitue une «totalité spatio-temporelle» (R. Bach, 1980). C'est pourquoi les thèses élaborées dans un passé récent ne sont plus pertinentes. L'ancien processus d'internationalisation est parvenu à un nouveau palier (M. Rochefort, 1998), et maintenant que le capitalisme a véritablement « atteint l'échelle mondiale » (O. Ianni, 1992) tous les pays deviennent solidaires. Comme l'affirme Octavio Paz (1990) : «Le nouveau n'est pas exactement le moderne, sauf s'il est porteur de la double charge explosive, à savoir : être la négation du passé et être l'affirmation de quelque chose de différent. »C'est le cas actuel de la globalisation. Nous sommes en effet témoins d'une période où des progrès spectaculaires, dus à l'alliance entre science et technologie ont le pouvoir de "transformer pour toujours les bases matérielles de notre vie, à une échelle qui était inconcevable il y a à peine un demi-siècle » (G. Barraclough, 1980).

Si le nouveau mode de production est global, c'est parce qu'il est aussi un mode de production scientifique et technique. Cette interdépendance entre science et technique, aujourd'hui sous la domination de la technique, se vérifie dans tous les aspects de la vie. Elle se rencontre dans toutes les parties du monde et elle constitue avec l'information la nouvelle variable motrice, qui permet aussi bien d'identifier un nouveau système temporel que l'organisation d'un nouvel espace.

Trois données constitutives de notre époque, à la fois cause et effet les unes des autres et solidaires à l'échelle mondiale, méritent alors d'être prises en compte: l'unicité technique, avec l'universalité des techniques, la convergence des moments avec la perception universelle de la simultanéité, et l'unité du moteur de la vie sociale avec l'universalisation de la plus-value.

15 Par unicité technique, nous entendons le fait que les techniques actuelles forment un système à l'échelle du globe, chaque lieu en abritant des fragments ou des pièces interdépendantes (G. Simondon, 1958). Tous les sous-espaces prennent part à cette "planétarisation de la technique ", même si sa diffusion s'effectue selon une intensité et des degrés de complexité différents.

16 Auparavant, les systèmes techniques n'étaient que locaux ou régionaux. À l'aube de l'histoire, il y avait autant de systèmes techniques que de lieux. Quand ils présentaient des similitudes, il n'y avait pas de contemporanéité entre eux et encore moins d'interdépendance fonctionnelle. L'histoire humaine est également celle de la diminution progressive du nombre de systèmes techniques sur la face de la terre. Le mouvement d'unification, accéléré par le capitalisme, atteint aujourd'hui son apogée, avec la prédominance, partout, d'un unique système technique, base matérielle de la globalisation.

17 Examinons maintenant, la convergence des moments. Durant des millénaires, l'histoire de l'homme s'est faite à partir de temps divergents, telle une somme de devenirs épars, disparates, incohérents. En revanche, celle de notre génération est celle où les moments ont convergé, ce qu'il advient de chaque lieu pouvant être immédiatement communiqué à n'importe quel autre, grâce à cette unification du temps et de l'espace à l'échelle planétaire.

18 L'instantanéité de l'information rapproche les lieux, rend possible une prise de connaissance immédiate d'événements simultanés et crée entre lieux et événements 
une relation unitaire à l'échelle du monde. Aujourd'hui, chaque moment comprend, partout, des événements qui sont interdépendants et inclus dans un même système global de relations.

Les progrès techniques qui, par l'intermédiaire des satellites, permettent de photographier la planète, nous permettent également d'avoir une vision empirique de la totalité des objets installés sur la face de la terre. Comme les photographies se succèdent à intervalles réguliers, nous obtenons une photo de l'évolution même du processus d'occupation de la croûte terrestre. La simultanéité photographiée est un fait véritablement nouveau et révolutionnaire pour la connaissance du réel et la représentation qu'en ont les sciences de l'homme.

La connaissance empirique de la simultanéité des événements et la signification de leur interdépendance constituent les bases de l'empirisation de l'universalité (Santos, 1984) et un facteur déterminant de la réalisation historique. Les acteurs hégémoniques de la vie économique, sociale et politique, peuvent choisir les lieux les plus appropriés à leurs agissements.

21 L'unité du moteur de la vie sociale se vérifie à partir de cette trame. Un marché global s'impose fondé sur l'échange global et sur une loi de la valeur universelle (T. dos Santos, 1993). On peut, de cette façon, parler d'une plus-value au niveau mondial, assurée par l'action convergente des grandes firmes, qu'elles soient privées ou publiques, nationales ou supranationales. Cette plus-value, devenue mondiale par la production et unifiée par le système financier, constitue le moteur de la vie économique et sociale sur toute la planète.

Les principaux vecteurs de ce processus sont les entreprises multinationales et les banques transnationales. Dans une situation de compétitivité, la recherche individuelle du plus grand profit possible n'a d'autre frontière que la capacité même de créer et d'utiliser des innovations productives et organisationnelles. À chaque moment, la plus forte des plus-values cherche encore à se dépasser elle-même.

Ironie suprême: cette plus-value si fugace ne peut pas être mesurée; alors qu'elle devient le principal levier, voire l'unique moteur des actions les plus caractéristiques de l'économie globalisée.

Unicité technique, convergence des moments et unicité du moteur sont des réalités dont l'interdépendance assure la production de cette intelligence planétaire qui est le support du processus actuel de globalisation.

Mais le phénomène technique, qui est à la racine de ces grandes transformations, a été insuffisamment utilisé comme point de départ de l'explication géographique.

Le milieu technico-scientifico-informationnel

De même qu'Olivier Buchsenschutz (1987) regrette que les archéologues ne se penchent que rarement sur les problèmes technologiques (entendons par là les traces matérielles laissées par les activités humaines), François Sigaud (1981) s'interroge à son tour sur la raison pour laquelle « les géographes évitent systématiquement l'étude des techniques, qui sont au centre des relations société-milieu ». On dira que cette critique est quelque peu injuste, puisque le thème des techniques apparait chez certains auteurs. Mais il est rare que le phénomène technique bénéficie d'un effort de généralisation, et soit abordé de façon systématique en tant que donnée explicative capable de servir à l'élaboration d'une théorie ou d'une épistémologie de la géographie. La philosophie des techniques, 
que chacun appelle de ses vœux, aurait beaucoup à gagner, et la reconstruction de la théorie sociale se verrait dotée d'une nouvelle version.

L'idée même de milieu géographique est inséparable de la notion de système technique. Nous pouvons admettre que l'histoire du milieu géographique a connu trois étapes : le milieu naturel, le milieu technique, le milieu technico-scientifico-informationnel.

Quand tout était milieu naturel, l'homme choisissait dans la nature ce qu'il considérait comme fondamental à l'exercice de la vie. Valorisées de façon variable selon les lieux et les cultures, ces conditions naturelles constituaient la base matérielle de l'existence du groupe. Depuis la fin du XVIII ${ }^{e}$ siècle, la mécanisation du territoire s'est développée et l'espace s'est densifié avec la présence des techniques, de la machine. On peut dire, avec Max Sorre (1948) et André Siegfried (1955) que c'est le moment où le milieu technique se surajoute en de nombreux lieux au milieu naturel, en cherchant à le remplacer. Aujourd'hui, il ne s'agit déjà plus de milieu technique, quand nous nous référons aux manifestations géographiques découlant des nouveaux progrès. Nous sommes face à la production de quelque chose de nouveau, que nous appelons milieu technico-scientificoinformationnel.

De la même façon, qu'elles contribuent à la création de nouveaux processus vitaux ou à la production de nouvelles espèces (animales et végétales), la science et la technologie alliées à l'information, sont à la base même de la production, de l'utilisation et du fonctionnement de l'espace et tendent à constituer son substrat.

Un véritable technocosme a vu le jour, une situation où la nature naturelle tend à reculer, parfois brutalement. L'idée d'un milieu artificiel, avancée par

31 A. Labriola en 1896 dans son étude intitulée Del Materialismo Stórico devient une évidence. La technique, en produisant un espace chaque fois plus dense devient le milieu d'existence d'une bonne partie de l'humanité (N. Rotenstreich).

Nous pouvons alors parler de scientifisation et de technicisation du paysage. D'un autre côté, l'information n'est pas seulement présente dans les choses, dans les objets techniques qui forment l'espace. Elle est aussi nécessaire à l'action réalisée sur ces choses; elle est le vecteur fondamental du processus social et les territoires sont équipés pour en faciliter la circulation.

Les espaces ainsi requalifiés répondent surtout aux intérêts des acteurs hégémoniques de l'économie, de la culture et de la politique, et sont pleinement incorporés aux nouveaux courants mondiaux. Le milieu technico-scientifico-informationnel est le visage géographique de la globalisation.

En même temps que grandit l'importance des capitaux fixes (routes, ports, silos, terre labourée, etc.) et des capitaux constants (machinerie, véhicules, semences spécialisées, fertilisants, pesticides, etc.), augmente aussi le besoin de mouvement, car le nombre et l'importance des flux va croissant - flux financiers inclus - et donne un relief particulier à la vie de relations.

35 Les équilibres préexistants sont rompus et de nouveaux s'imposent, du point de vue de la quantité et de la qualité de la population et de l'emploi, des capitaux utilisés, des formes d'organisation, des relations sociales, etc. Conséquence plus strictement géographique, l'arène de la production diminue, tandis que l'aire correspondante s'accroît. L'espace réservé au processus direct de la production se restreint, tandis que s'agrandit l'espace des autres instances de la production : circulation, distribution et consommation. 
Cette réduction de l'arène nécessaire, par unité de temps et de superficie, à la production des mêmes quantités, avait été prévue par Marx, qui avait appelé ce phénomène réduction de l'arène. Le processus de spécialisation, en créant des aires distinctes où la production de certains produits est plus avantageuse, augmente le besoin d'échanges qui se feront dans des espaces plus vastes, phénomène que Marx a intitulé agrandissement de l'aire.

Les possibilités techniques et organisationnelles de transfert à distance des produits et des ordres, ont pour résultat que ces spécialisations productives sont solidaires au niveau mondial. Quelques lieux tendent à se spécialiser à la campagne comme à la ville, et cette spécialisation est davantage due aux conditions techniques et sociales qu'aux ressources naturelles.

Comme on produit chaque fois plus de valeurs d'échange, la spécialisation ne tarde pas à impliquer davantage de circulation, et le rôle de celle-ci, dans la transformation de la production et de l'espace, devient fondamental. Une de ses conséquences est en effet l'approfondissement des spécialisations productives qui tendent, à leur tour, à susciter plus de circulation. Ce cercle vicieux - ou vertueux ? - dépend de la fluidité des réseaux et de la flexibilité des règlements.

9 La dynamique des espaces de globalisation suppose une adaptation permanente des formes et des normes. Les formes géographiques, c'est-à-dire les objets techniques requis pour optimiser une production, n'autorisent cette optimisation qu'au prix de l'instauration et de l'application de normes juridiques, financières et techniques, adaptées aux nécessités du marché. Celles-ci sont créées à différents niveaux géographiques et politiques, mais en vue de la compétitivité mondiale; les normes globales, induites par les organismes supranationaux et le marché, tendent à configurer les autres. Et les normes du marché tendent à configurer les normes publiques.

Les nouveaux sous-espaces sont plus ou moins capables de rentabiliser la production. Chaque combinaison a sa propre logique et autorise des formes d'action spécifiques de la part des agents économiques et sociaux. Les actions hégémoniques s'établissent et se réalisent par l'intermédiaire d'objets hégémoniques. Comme dans un système de systèmes, le reste de l'espace et le reste des actions sont appelés à collaborer. Nous pouvons donc parler de productivité spatiale ou de productivité géographique, notion qui s'applique à un lieu, mais en fonction d'une activité ou d'un ensemble d'activités. Cette catégorie se réfère davantage à l'espace productif, c'est-à-dire au travail de l'espace. Sans minimiser l'importance des conditions naturelles, ce sont les conditions artificiellement créées qui ressortent, en tant qu'expression des processus techniques et des supports géographiques d'information.

Serions-nous face à un déterminisme de type nouveau, un néo-déterminisme de l'espace artificiel?

Comme dans les périodes antérieures, il y a inégalité selon les continents, les pays et à l'intérieur de chaque pays. Dans certains cas, comme en Europe occidentale, la plus grande partie des territoires nationaux est occupée par ce milieu technico-scientificoinformationnel. Dans d'autres cas, comme au Brésil, il recouvre une vaste étendue, mais il est loin de couvrir la totalité du territoire. Dans la majeure partie des pays, il se limite à peine à des taches ou à des points.

Horizontalités, verticalités, réseaux, région 
Dans les conditions actuelles, les agencements spatiaux ne se font pas seulement comme il en était dans les régions du passé, à travers des figures formées de points continus et contigus. À côté de ces taches, ou par dessus, il y a également des constellations de points discontinus, mais liés entre eux, qui définissent un espace de flux régulateurs. Tout cela forme un espace. C'est à partir de ces nouvelles subdivisions que nous devons envisager de nouvelles catégories analytiques.

Les segmentations actuelles de l'espace suggèrent que l'on admette deux coupes appelées ici horizontalités et verticalités. D'un côté, il y a des étendues continues formées de points qui se regroupent sans discontinuité, comme dans la définition traditionnelle de région. Ce sont les horizontalités. D'un autre côté, il y a des points qui, séparés les uns des autres, assurent le fonctionnement global de la société et de l'économie. Ce sont les verticalités.

Dans le premier cas, la solidarité entre les éléments formateurs est surtout due au processus direct de la production. Il en est ainsi des relations villes/campagnes où l'attraction entre sous-espaces ayant des fonctionnalités différentes correspond à une production proprement dite, puisque la ville, surtout dans les zones les plus touchées par la modernité, est le lieu de régulation du travail agricole. Dans le second, les relations interurbaines par exemple, la solidarité est obtenue par le biais de la circulation, de l'échange et de leur régulation.

L'idée et la réalité des réseaux sont donc des données fondamentales pour comprendre la dynamique actuelle des territoires. Notion considérée comme éminemment géographique, elle peut être appréhendée selon au moins trois sens, comme le propose H. Bakis (1993) : a) polarisation de points d'attraction et de diffusion : cas des réseaux urbains ; b) projet abstrait : cas des méridiens et des parallèles dans la cartographie du globe ; c) projection concrète de lignes de relations et de liaisons : cas des réseaux hydrographiques et des réseaux techniques territoriaux, mais aussi des réseaux de télécommunications hertziennes, malgré l'absence de lignes et une structure physique limitée aux nœuds.

Qu'est-ce qu'un réseau ? Les définitions se multiplient, mais on peut admettre qu'elles se regroupent en deux catégories: celle qui ne prend en compte que sa réalité matérielle, attitude qui conduit à une définition formelle que N. Curien (1988) illustre de la façon suivante: "Toute infrastructure permettant le transport de matière, d'énergie ou d'information qui s'inscrit sur un territoire où elle se caractérise par la topologie de ses points d'accès ou points terminaux, ses segments de transmission, ses nœuds de bifurcation ou de communication. »; celle qui inclut la dimension sociale, ce qui paraît plus juste.

Compte tenu des personnes, des messages et des valeurs qui le sillonnent, tout réseau est en effet social et politique, sans quoi, malgré la force avec laquelle la matérialité s'impose à nos sens, il ne serait qu'une simple abstraction. La notion d'espace maillé vient de cette construction de l'espace comme cadre de vie, prêt à répondre aux encouragements de la production sous toutes ses formes matérielles et immatérielles. C'est sans doute pour cela qu'O. Dollfus (1971) propose que le terme de réseau soit limité aux systèmes créés par l'homme, laissant aux systèmes naturels le nom de circuit.

Les réseaux sont porteurs d'informations sous la forme de produits, de marchandises, d'idées, d'argent, de messages affectifs. Leur fonction fondamentale est d'assurer des 
liaisons, dans leurs aspects les plus divers, et leur force est d'autant plus grande qu'est nombreuse la variété des communications que leur contenu technique rend possible.

Ils sont à la fois globaux et locaux; globaux car ils recouvrent tout l'écoumène et constituent le principal instrument d'unification de la planète ; locaux, puisque chaque lieu, à travers sa structure technique et informationnelle, accueille une fraction, plus ou moins grande, des réseaux globaux. À l'échelle du lieu, ils servent au travail et au capital productif et en déterminent la nature. Nationaux ou mondiaux, ils président à la division internationale du travail et aux formes de coopération (M. L.Silveira, 1994).

51 Grâce aux progrès techniques et aux formes actuelles de réalisation de la vie économique, les réseaux productifs commerciaux, de transport, d'information tendent toujours à être plus globaux. B. Kayser et A. Brun (1993) montrent comment «l'espace rural même dans ses zones apparemment marginales est complètement intégré au système socio-économique global ». Mais la forme la plus achevée et la plus efficace du réseau est due à l'activité financière en raison de la fluidification de l'argent et à son utilisation instantanée et généralisée.

Les réseaux sont incompréhensibles, si nous ne les regardons qu'à partir de leurs manifestations locales ou régionales. Mais celles-ci sont également indispensables pour comprendre comment ils fonctionnent à l'échelle du monde car, à partir d'un mouvement privilégié que nous souhaitons mettre en lumière, nous pouvons découvrir le mouvement global à travers des mouvements particuliers. En effet, « tous ces cycles sont synchrones; ils coexistent, se mêlent, ajoutent leurs mouvements ou les retranchent des oscillations de l'ensemble » (F. Braudel, 1979).

L'espace est le théâtre de tout un ensemble d'objets et de flux ayant différents contenus, intensités et orientations. Ceux-ci forment des réseaux inégaux aux caractéristiques diverses, qui se superposent et s'enchevêtrent à différents niveaux et échelles pour constituer l'espace banal, c'est-à-dire l'espace de tous les hommes, de toutes les entreprises, de toutes les organisations, de toutes les actions, en un mot, l'espace géographique.

54 L'utilisation de cet espace est sélective et ce qu'on appelle de façon abusive espace de flux n'est en réalité rien d'autre qu'un sous-système de l'espace total, formé d'objets dotés d'un niveau supérieur de technicité et d'actions marquées par un niveau supérieur d'intentionnalité et de rationalité. Ce sont des objets et des actions dans lesquels le contenu des informations est plus dense que dans d'autres sous-systèmes $d u$ même espace.

Seuls les acteurs hégémoniques se servent de tous les réseaux et utilisent tous les territoires. Mais l'espace réticulaire est celui de leur choix. Voilà pourquoi les territoires nationaux se transforment en un espace national de l'économie internationale et les systèmes d'ingénierie les plus modernes créés dans chaque pays sont mieux utilisés par des firmes transnationales que par la société nationale ellemême.

D'où la mention fréquente d'un espace sans frontières et d'un "capitalisme sans frontières » (P. Ciccolella, 1993), où les entreprises multinationales court-circuitent les États (R. Petrella, 1989) et où ce sont les affaires et non pas les gouvernements qui gouvernent. Cependant, croire que l'État est devenu inutile est une erreur. L'émergence d'organisations et d'entreprises multinationales renforce au contraire le rôle de l'État, devenu plus indispensable qu'auparavant (Groupe de Lisbonne, 1994). 
57 Dans ce même courant post-moderniste, la négation de l'idée de région trouve également sa place. C'est un fait que le phénomène a fondamentalement changé de sens, mais on ne peut déclarer qu'il a cessé d'exister.

Nous sommes loin de cette solidarité organique qui fut au cœur même de la définition du phénomène régional. Ce que nous avons aujourd'hui devant nous, ce sont des solidarités organisationnelles mais les régions existent car elles sont soumises à des agencements organisationnels, créateurs d'une cohésion basée sur des rationalités d'origines lointaines qui deviennent le fondement de leur existence. Que reste-t-il, dans ces conditions, des anciennes définitions de la région?

59 Au cours de l'histoire, les régions se sont formées à travers la territorialité d'un groupe, d'où provenaient les caractéristiques de son identité, de son exclusivité et de ses frontières. La différence entre les aires était due à cette relation directe avec l'environnement, c'est-à-dire à la seule présence de ce groupe, sans autre médiation. Nous pouvons dire que la solidarité caractéristique de la région se faisait alors, presque exclusivement, en fonction des arrangements locaux. D'où la confusion faite parfois, aux premiers temps de la géographie scientifique, entre les notions de région et de paysage.

Les transformations mondiales de ce siècle, qui se sont accélérées de façon vertigineuse dans l'après-guerre, ont conduit à l'effondrement de cette configuration régionale du passé. Certains parlent d'ailleurs de la disparition de la région, tant est grande la difficulté d'appréhender les nouvelles constructions régionales. On dit aujourd'hui que le temps a effacé l'espace. De la même façon, on affirme que l'expansion de la présence du capital hégémonique, élimine les différenciations régionales et empêche même de penser en termes de région.

61 On opposera à ces affirmations qu'à l'époque actuelle les lieux sont toujours plus la condition et le support des relations globales qui, sans eux, ne se feraient pas. Les régions sont devenues des lieux fonctionnels du Tout, des espaces de convenance, une particularité, ce qui, chez Lukacs (1970), signifie " un champ de médiations ».

62 Avec l'essor de la division internationale du travail et l'augmentation exponentielle des échanges, on assiste ainsi parallèlement à une accélération du mouvement et à des changements de plus en plus fréquents, de la forme et du contenu des régions. Ceci ne les élimine pas. Mais elles se transforment de façon continue, ce qui leur concède une moindre durée.

63 Nous nous étions habitués à l'idée que la région était un sous-espace élaboré sur le temps long; cependant ce qui fait la région, ce n'est pas la longévité de l'édifice, c'est sa cohérence fonctionnelle et c'est en cela qu'elle se distingue ou non de ses entités congénères. Le fait d'avoir une vie courte change la définition de la coupe territoriale sans pour autant la supprimer.

$64 \mathrm{Au}$ contraire, l'épaisseur de l'actualité est accrue et du fait du plus grand volume d'événements par unité d'espace et par unité de temps, la région continue à exister, mais à un niveau de complexité jamais vu par l'homme.

65 Aujourd'hui, aucun sous-espace de la planète ne peut échapper au processus conjoint de globalisation et de fragmentation, c'est-à-dire d'individualisation et de régionalisation. La technologie elle-même " est implosive dans ses effets » (D. Schon, 1971, 1973), et l'accélération du temps, en accentuant la différenciation des événements, augmente la différenciation entre les lieux, tandis que le phénomène de la 
région gagne en universalité. En s'étendant sur tout l'Ecoumène, il tend à le rediviser entièrement.

En un mot, nous avons cheminé, au long des siècles, de l'antique communion individuelle des lieux avec l'univers à la communion globale actuelle : l'interdépendance universelle des lieux est la nouvelle réalité du territoire.

Technique, information, communication : la typologie des sous-espaces

L'espace géographique étant formé par un ensemble indissociable de systèmes d'objets et de systèmes d'actions, chaque sous-espace inclut une partie de ces sous-systèmes.

Chaque lieu, chaque sous-espace se définit aussi par la présence conjointe, indissociable, d'une technosphère et d'une psychosphère qui fonctionnent de façon unitaire. La technosphère est le monde des objets, la psychosphère est la sphère de l'action. Et les objets, naturels ou artificiels, sont hybrides - au sens proposé par B. Latour (1991) et A. Gras (1993) - puisqu'ils n'ont plus d'existence réelle, valorisatrice, sans les actions. Ainsi, chaque lieu, chaque sous-espace, se définit-il autant par son existence corporelle que par son existence relationnelle. D'ailleurs, c'est ainsi que les sous-espaces existent et se différencient les uns des autres.

Les espaces de la globalisation présentent des charges différentes de contenu technique, de contenu informationnel et de contenu de communication. Les lieux se définissent donc par la densité de ces différents attributs qui s'interpénètrent et dont la fusion les caractérise et les distingue.

70 La densité technique est donnée par les divers degrés d'artifice. Les situations limites seraient, d'un côté, une aire naturelle jamais touchée par l'homme - une écologie sauvage - et, de l'autre, une aire où la matérialité est formée de ce que Simondon (1958) a appelé « objets techniques concrets", comme dans le centre d'affaires d'une grande ville où des espaces intelligents sont disposés pour répondre immédiatement aux desseins de ceux qui les ont conçus et produits, objets bien plus parfaits que la nature elle-même.

71 La densité informationnelle dérive en partie de la densité technique. Les objets techniques, riches en information, peuvent néanmoins ne pas être activés, restant à l'état de repos, dans l'attente d'un acteur. L'information ne se parachève en effet qu'avec l'action. La densité informationnelle nous indique le degré d'extériorité du lieu et sa propension à entrer en relation avec d'autres lieux, mais ses implications sont fonction de secteurs et d'acteurs privilégiés. L'information univoque, obéissant aux règles d'un acteur hégémonique, introduit dans l'espace une intervention verticale, qui ignore généralement celui qui l'entoure et se met au service de celui qui détient les leviers de commande.

72 La densité de communication résulte de ce que G. Berger a appelé le «caractère humain du temps de l'action ", car l'événement peut être vu comme praxis intersubjective (J. L. Petit, 1991) ou praxis transindividuelle (Simondon, 1958). Ce temps pluriel du quotidien partagé est le temps conflictuel de la co-présence. Comme lieu de l'avenir solidaire, cet espace banal de la géographie (et non pas l'espace spécial, particulier, déterminé de l'économiste, de l'anthropologue, du psychologue ou encore de l'architecte ou du philosophe) est créateur de l'interdépendance et de la solidarité générées par les situations de face à face qu'évoque Schutz (1967). Pour ce résultat, l'essentiel est que « toi et moi ayons le même cadre », puisque « il n'y a que dans cette situation [...] que je peux assurer avec plus ou moins de certitude, à l'intérieur de la réalité directement 
vécue (dont j'ai fait l'expérience) que la table que je vois est la même, et la même dans toutes les situations envisagées ».

Les relations techniques et informationnelles peuvent être indifférentes à l'environnement social, mais les relations de communication en sont une résultante. Les deux premières sont plus dépendantes de la sphère de la matérialité, de la technosphère, les autres le sont davantage de la psychosphère, même si, dans tous les cas, technosphère et psychosphère interagissent. Mais, les relations de communication, générées dans le lieu ont, toutefois, plus que les autres, une geographic flavour, en dépit de l'origine, peut-être, lointaine des objets, des hommes et des ordres qui les font se mouvoir.

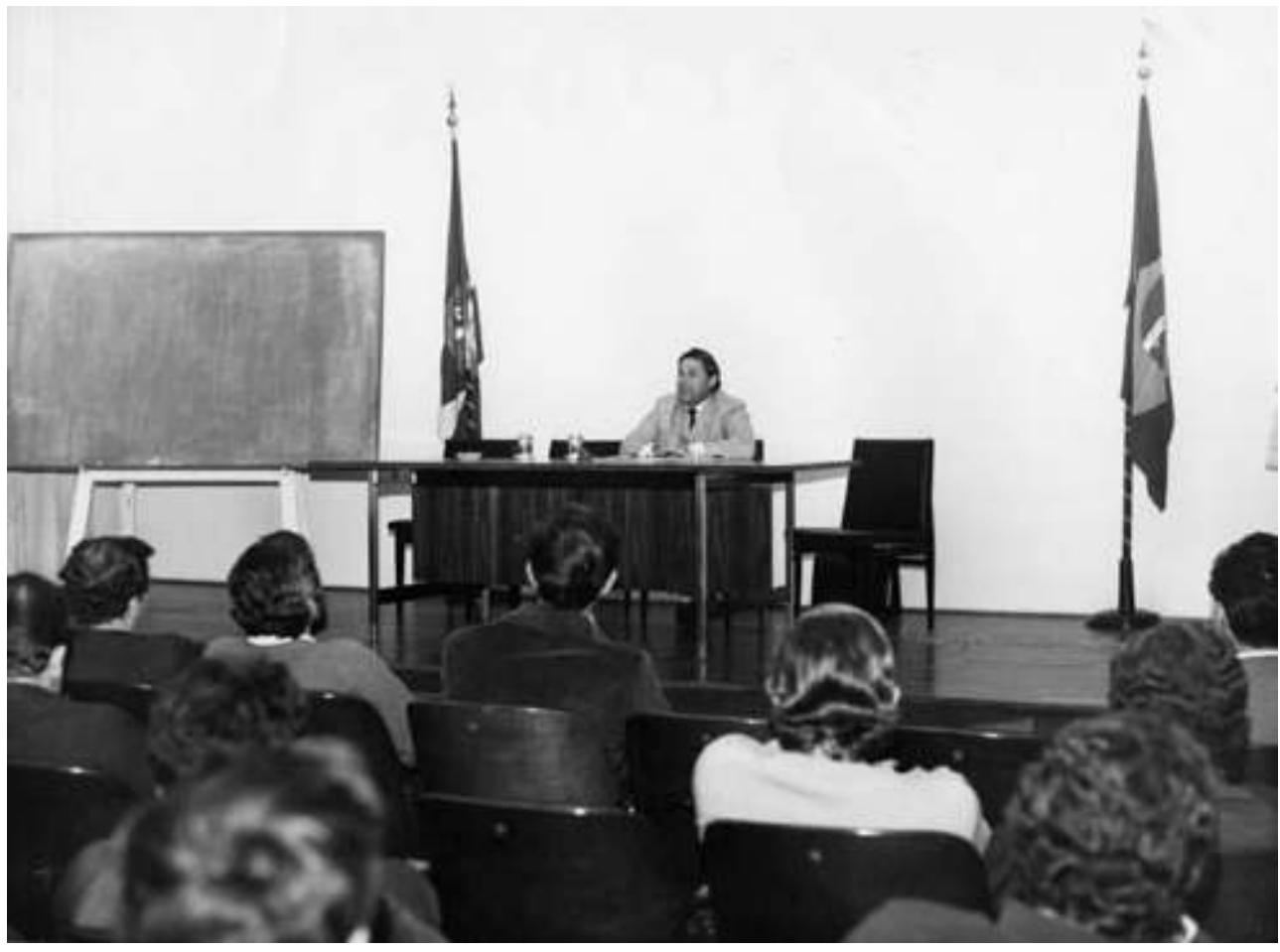

Conférence à Floriano, Brésil (1971)

\section{BIBLIOGRAPHIE}

BACH, R. 1980. " On the holism of a world-system perspective ", in HOPKINS, T.K. and I. WALLERSTEIN (eds.) Processes of the World-System. Beverly Hills, Sage, 289-310.

BAKIS, H. 1993. Les Réseaux et leurs enjeux sociaux. Paris, Presses Universitaires de France (Que saisje ?).

BARRACLOUGH G., 1980. Tendances actuelles de l'histoire mondiale, Flammarion.

BERGER, G. 1964. Phénoménologie du temps et prospective. Paris, Presses Universitaires de France. 
BRAUDEL, F. 1979. Le Temps du Monde. T. III. Civilisation Matérielle, Économie et Capitalisme, XVXVIIIe siècle. Paris, Armand Colin.

BUCHSENSCHUTZ, O. 1987. « Archéologie, typologie, technologie », Techniques et Cultures, 9, 17-26. CICCOLELLA, P. 1993. « Hacia un capitalismo sin fronteras ? o La Historia recién comienza », (mimeo, 13 p.).

CURIEN, N. 1988. « D'une problématique générale des réseaux à l'analyse économique du transport des informations ", in G. DUPUY. Réseaux territoriaux, Caen, Paradigme, 211-228. DOLLFUS, O. 1971. L'Analyse géographique. Paris, Presses Universitaires de France.

DOS SANTOS, T. 1993. "Quelques idées sur le système monde », Cahier du Gemdev 20, 55-66. DURAND, M. F. ; LÉVY, J. ; RETAILLÉ, D. 1992. Le Monde, espaces et systèmes. Paris, Presses de la Fondation Nationale des Sciences Politiques et Dalloz.

GRAS, A. 1993. Grandeur et dépendance. Sociologie des macro-systèmes techniques. Paris, Presses Universitaires de France.

GROUPE DE LISBONNE. 1995. Limites à la compétitivité, pour un nouveau contrat mondial. Paris, La Découverte.

IANNI, O. 1992. A Sociedade Global. Rio de Janeiro, Civilização Brasileira.

KAYSER, B. et A. BRUN. 1993. La place de l'espace rural sans une politique d'aménagement du territoire, (mimeo 6 p.) (6 juillet 1993)

LABRIOLA, A. 1896. «Del materialismo stórico » in A. LABRIOLA (1947), La concezione materialista della storia. Bari.

LATOUR, B. 1991. Nous n'avons jamais été modernes. Essai d'anthropologie symétrique. Paris, Éditions La Découverte.

LUKACS, G. 1970. Introdução a uma estética marxista. Rio de Janeiro, Civilização Brasileira.

PAZ, O. 1990. Los hijos del limo. Del romanticismo a la vanguardia. (1974). Barcelona, Seix Barral, 3e ed.

PETIT, J. L. 1991. «Oublier l'événement ? Le fait contre l'événement chez J.Bennett », in J. L. PETIT (org.). L'Événement en perspective. Paris, Éditions de l'École de Hautes Études en Sciences Sociales, 259-262.

PETRELLA, R. 1989. « La mondialisation de la technologie et de l'économie, une (hypo)thèse prospective », Revue Futuribles, 135, 3-25.

ROBERTSON, R. 1990. " Mapping the global condition : globalization as the central concept », Theory, Culture and Society, 7, 15-30.

ROCHEFORT, M. 1998, Redes e Sistemas. Ensinando sobre o Urbano e a Região, São Paulo, Hucitec.

SANTOS, M. 1984. " The rediscovery and the remodelling of the planet in the technico-scientific period and new roles of sciences », International Social Science Journal, 36, 4, Paris, Unesco.

SCHON, D. A. 1973. Beyond the Stable State: Public and Private Learning in a Changing Society. Harmondsworth, Penguin Books (1971).

SCHUTZ, A. 1967. The Phenomenology of the Social World. Evanston, Northwestern University, Illinois.

SIEGFRIED, A. 1955. Aspects du XX $X^{e}$ siècle. Paris, Hachette. 
SIGAUD, F. 1981. « Pourquoi les géographes s'intéressent-ils à peu près à tout sauf aux techniques ? », L'Espace Géographique, 4, 291-293.

SILVEIRA, M.L. (1994). »Os novos conteúdos da regionalização : lugares modernizados e lugares letárgicos no planalto nordpatagónico argentino », Finisterra, XXIX, 58, 65-83.

SIMONDON, G. 1989. Du mode d'existence des objets techniques. (1958, 1969). Paris, Aubier. (Édition augmentée d'une préface de J. Hart et d'une postface de Y. Deforge).

SORRE, M. 1950. Les Fondements de la géographie humaine. Paris, A. Colin. 